\title{
Application of the Hybrid CBL+TBL Method in Internal Medicine Teaching Practice of TCM Universities
}

\author{
Yuan Fang, Lijie Liu, Lihong Nie, Min Xiao, Qian Lin, Nan Guo, Xuebing Zhang, Shaodan Tian, \\ Mei Wang"
}

Dongzhimen Hospital, Beijing University of Chinese Medicine, Beijing, China

Email address:

13601382677@126.com (Mei Wang)

${ }^{*}$ Corresponding author

\section{To cite this article:}

Yuan Fang, Lijie Liu, Lihong Nie, Min Xiao, Qian Lin, Nan Guo, Xuebing Zhang, Shaodan Tian, Mei Wang. Application of the Hybrid CBL+TBL Method in Internal Medicine Teaching Practice of TCM Universities. International Journal of Chinese Medicine.

Vol. 5, No. 3, 2021, pp. 48-52. doi: 10.11648/j.ijcm.20210503.12

Received: November 14, 2021; Accepted: December 14, 2021; Published: December 24, 2021

\begin{abstract}
Internal medicine plays a critical role in the curriculum system of traditional Chinese medicine (TCM) universities, which is the main course in TCM colleges and the professional knowledge must be mastered by medical students. However, due to the immense contents and concepts in internal medicine, the traditional lecture-based learning (LBL) led to dissatisfactory education effects. The purpose of the study is to explore the application of hybrid case-based learning (CBL) + team-based learning (TBL) method in educational practice of internal medicine in TCM universities. The undergraduates majoring in Chinese medicine of grade 2014, 2015 and 2016 from Beijing University of Chinese Medicine (BUCM) were enrolled. Students of grade 2014 were utilized as the control group, adopting traditional teaching method. Students of grade 2015 and 2016 were employed as the experimental groups, using the hybrid CBL+TBL teaching method. At the end of the internal medicine curriculum, the teaching resultant was evaluated by both theoretical test and skill assessment. The results demonstrated that experimental groups scored better than control group in both theory and clinical examinations, and the discrepancies were significant, respectively. The introduction of hybrid CBL+TBL teaching method into education of internal medicine in TCM universities promoted students' ability of self-learning and sense of teamwork, so as improved the teaching effectiveness of clinical courses.
\end{abstract}

Keywords: Hybrid CBL+TBL Teaching Method, TCM Universities, Internal Medicine, Undergraduate Education

\section{Background}

Internal medicine is an important branch of clinical medicine, mainly dealing with the diagnosis and (nonsurgical) treatment of internal organ diseases, especially in adults. Internal medicine, with wide aspects and strong integrity, influences the development of medical science substantially. The goal of internal medicine education is to instruct the pathogenesis, diagnosis, examination and treatment of diseases not requiring surgery to medical students $[1,2]$.

Learning internal medicine well is essential for the cultivation of clinic ability and skill of a medicine student, which is also the basis to become a qualified doctor. However, for internal medicine, the magnitude of curriculum is extensive, and the proportion of concept is tremendous [3].
The traditional teaching method leads to boring class atmosphere and disappointing study consequence [4]. In addition, students majoring in traditional Chinese medicine (TCM) generally possess infirm knowledge and skill of Western medicine. Therefore, it is compelling to explore novel teaching method of internal medicine suitable for TCM universities and undergraduates.

In recent years, case-based learning $(\mathrm{CBL})$ and team-based learning (TBL) have been widely used in medicine education. CBL is a teaching method which applies typical cases to analyze theoretical concept and helps students to understand knowledge better [5]. TBL is a teaching method which takes teamwork as its core perception and stimulates students' interest through discussions within and between groups [6]. Hybrid CBL + TBL teaching method organically combines the two teaching methods, which gives full play to the guiding and 
monitoring role of teachers, cultivates the learning autonomy and creativity of students, and ultimately achieves the purpose to optimize internal medicine teaching effect [7]. This research adopted the hybrid $\mathrm{CBL}+\mathrm{TBL}$ method in the educational practice of internal medicine for undergraduates in the Dongzhimen Hospital of Beijing University of Chinese Medicine (BUCM) in order to bring the advantages of different teaching modes into full play and pave the way for further standardized education. The aim of this education method reform is to make the teaching and learning of clinic courses more efficient, and to find a new pathway suitable for teaching internal medicine in TCM colleges.

\section{Methods}

\subsection{Study Subjects}

The eight-year program medical students majoring in TCM of grade 2014, 2015 and 2016 from BUCM were selected as the research volunteers. The control group was composed by 85 students of grade 2014, involving 24 males and 61 females, aged from 21 to 23 years old. The experimental groups were consisted of 109 students in grade 2015 (38 males and 71 females) and 103 students in grade 2016 (41 males and 62 females), ranged from 21 to 23 years old. Because one student was on sick leave in the final exam, 102 were finally incorporated in grade 2016. All students have completed the three-year curriculum of basic medicine, and there is no significant disparity between the three groups in fundamental information.

\subsection{Methods}

The Internal Medicine issued by the people's medical publishing house was used as the textbook for every grade. The lecturers and assistants of the three classes were the same. The course contents included internal diseases from respiratory system, circulatory system, digestive system, urinary system, blood system, endocrine system, and nervous system, with the total lesson period reaching to 36 hours.

The traditional teaching mode was adopted for the control group. Firstly, teachers explained the key points of the theory content and prepared questions for students. Then students went to clinic departments for bedside inquiry and physical examination of patients. Finally, according to the condition of patients, teachers asked questions concerning the diagnosis, differential diagnosis, and treatment to train the basic capacity of medical students.

The hybrid CBL + TBL teaching method was applied for the experimental groups. Typical cases were selected by teachers before class and distributed to students as class preview. The analysis of clinic cases was performed using CBL teaching method. TBL teaching method was also introduced into the class and combined with CBL teaching method. Students were divided into groups containing about 10 peoples to prepare problems for class discussion and consider answers toward the case. Then, all the groups went to clinic departments to inquire and examine patients, and made diagnosis, differential diagnosis, and treatment plan through discussion within and between groups. Every student in the whole class shared out the work and cooperated with one another. Instead of receiving knowledge passively, students participated into the whole teaching activities, finding and learning knowledge by themselves. Finally, teachers gave answers to students' questions and offered summary comments on students' performance.

\subsection{Effect Evaluation}

At the end of the course, both control group and experimental groups participated in the theoretical examination and clinical assessment. The theoretical examination contained a set of questions with the total points of 100. Each student randomly selected a case analysis in question bank system for the clinical assessment, giving answers to questions concerning the diagnosis, differential diagnosis, supplementary examination and treatment measures. The total points for the clinical assessment were 10 . The test scores of all students were documented for further statistical analysis.

\subsection{Data Analysis}

Statistical analysis was performed utilizing GraphPad Prism 8 (GraphPad Software Inc., La Jolla, CA). All data were presented as the mean \pm standard error of mean (mean \pm SEM). Nonparametric Kruskal-Wallis test was used to compare differences between groups, followed by a false discovery rate (FDR) correction for multiple comparisons. Bilateral $P$ values less than 0.05 were identified as statistically significant.

\section{Results}

\subsection{Comparison of Theoretical Examination Results}

The traditional teaching method was applied to students majoring in TCM in grade 2014. The average score of theoretical exam was $72.910 \pm 0.986$. Students majoring in TCM from grade 2015 and 2016 employed the hybrid CBL + TBL teaching mode. The average score of theory examination was $77.030 \pm 0.957$ and $75.260 \pm 1.186$, respectively. Compared with the students of grade 2014, students from grade 2015 and 2016 performed better in the theoretical test, and the results were statistically significant $(P=0.0126$ and 0.0388 , respectively) (Figure 1).

\subsection{Comparison of Clinical Assessment Results}

The average clinical assessment score of 2014 grade was $7.271 \pm 0.135$. The average point for students from grade 2015 was 7.904, with a SEM of 0.124 . The mean of grade 2016 in clinical test was 7.887, and the SEM was 0.099. Compared with the students of grade 2014, students of grade 2016 scored higher in clinical assessment, and the difference was significant $(\mathrm{P}<0.0001)$. Students from grade 2015 performed better than grade 2014, and the discrepancy was significant $(\mathrm{P}=0.0003)$ (Figure 2). 


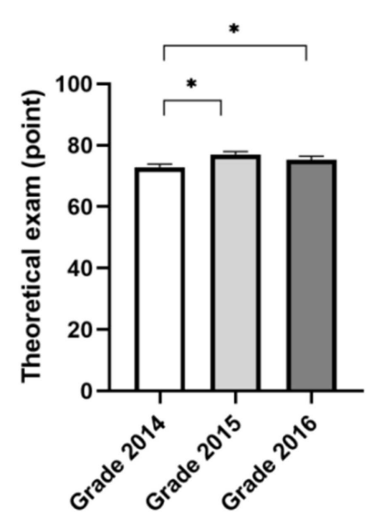

Figure 1. Comparison of theoretical examination results. $* P<0.05$.

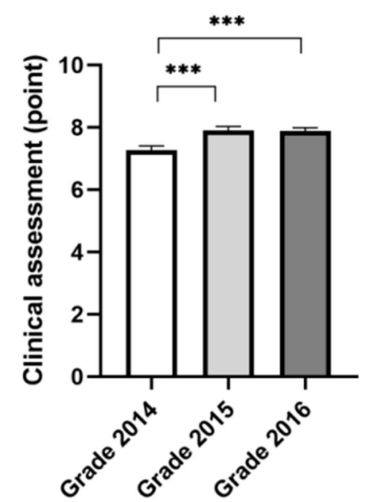

Figure 2. Comparison of skill assessment results. $* P<0.05$.

\section{Discussion}

The standards of undergraduate medical education-clinical medicine specialty (trial) jointly issued by the ministry of education and the ministry of health clearly putted forward that medicine colleges must actively carry out the reform of education methods with "student-centered" and "autonomous learning" as the main contents. The goal of medicine education is to cultivate students' clinical thinking and make them qualified doctors [8]. Therefore, in the "post-era" stage of teaching reform, how to improve students' clinical thinking and promote their comprehensive quality is one of the key points in teaching method modification [9].

Internal medicine is not only the fundamental courses of medicine colleges, but also the indispensable part of undergraduate curriculum in TCM universities. The contents of internal medicine possess universal significance in the theory and practice of clinical medicine education [10]. In the past, the teaching of internal medicine in TCM colleges mainly employed the lecture-based learning (LBL) method, with teachers as the principal part in teaching practice [11]. In this research, in accordance with the curriculum and examination requirement of internal medicine for TCM students, teachers educated the diagnosis, differential diagnosis, and treatment measures of diseases through CBL+TBL teaching method, expecting to find a new method suitable for the education of internal medicine in TCM universities.

In the current study, CBL teaching approach was utilized to cultivate students' ability of clinical reasoning and autonomous learning. Teachers have made adequate preparations and discussions in case selection, medical record collection and instruction method standardization. A randomized control trial published in 2020 demonstrated that, in contrast to LBL, CBL group responded better to questions relating to orthodontic diagnosis, indicating that $\mathrm{CBL}$ is an effective and acceptable teaching approach [12]. After case analysis, practical manipulation was carried out. TBL teaching mode was employed in current research to train students' basic clinical skills such as inquiry and physical examination in form of team cooperation. Group discussion further strengthened students' clinical intellection, deepened their impression, and improved the learning efficiency. Previous study performed by Rezende et al. revealed that TBL was an efficient approach associated with better acceptance and higher motivation of students [13]. TBL application in Chinese medical education is limited [14], and our investigation concerning TBL utilization in TCM university facilitates wider application of this methodology in medical education of China. One of the hot points in educational reform of medical colleges is how to cultivate undergraduates' ability and quality, which is more important than the simple instruction of knowledge [15]. Although the hybrid CBL + TBL teaching procedure has been applied among basic medicine and clerkship disciplines with promising outcomes [16], its usage in TCM education has not been reported according to our knowledge. In our study, students possessed the initiative of learning and teachers played the role of guidance, such interaction fully mobilized students' learning enthusiasm and deepened their mastery of literacy. The hybrid teaching method cultivates more qualified TCM doctors in limited class hours and space, enhances the competitiveness of graduates from TCM colleges in job application, and contributes to the improvements of TCM education.

Present results showed that the hybrid CBL + TBL teaching method performed better than the traditional teaching method. The advantages of CBL+TBL are as follows: (1) Typical cases from clinical departments provided operative materials for students to learn autonomously, promoted their subjective initiative and facilitated their learning of professional lessons. In addition, in the process of pre-class study and classroom discussion, the ability of analysis and expression were improved through mutual learning and communication between students and teachers, which further developed the motivation and efficiency of students' autonomous learning [17]. (2) Through the investigation and discussion concerning typical cases, the students' research enthusiasm and eagerness have been significantly triggered, giving full play to the role of "student-centered" education reform, which is of positive significance to students' theoretical learning and clinical diagnosis thinking ability [18]. (3) In the bedside instruction, the team members not only divided the work but also cooperated with each other. The interrogation and physical examination of actual patients, combined with the summarization and discussion of cases, facilitated students to 
immerse themselves in the classes. The bedside teamwork is not "the empty phrases on a scrap of paper", but the practical method enhanced students' learning enthusiasm and teamwork capability [19]. Therefore, under the hybrid teaching mode, students carried out appropriate pre-class learning, made sufficient preparations for classroom discussion, and eventually improved learning efficiency and teaching effectiveness. The hybrid TBL + CBL teaching method is based on teamwork, combined theory with practice, which has the obvious advantages in cultivating students' ability of active learning, critical thinking and team cooperation spirit.

At the same time, we also observed that although students from grade 2015 and 2016 had superior performance in theory and practice compared with students from grade 2014, students of grade 2015 acquired higher scores than grade 2016. The main reason for the phenomenon might be attributed to the homogenization of education. In a recent study published in 2020, researches homogenized the teaching of indications and technique of digital rectal examination, improved the skill training, and achieved greater satisfaction from students [20]. In our study, the lecturers or tutors teaching grade 2015 were more homogenous and had more lecturing experience, intimating that more unified and standardized education process brings out more encouraging outcomes. The results suggest that hybrid CBL+TBL method combined with standardized knowledge instruction and bedside tutoring promote the homogenization of medical education.

Through applying the hybrid CBL+TBL education method in BUCM, both theoretical study and clinical capacity of TCM students improved significantly, demonstrating the feasibility of implementing the current teaching approach in TCM universities.

\section{Conclusion}

In summary, through the practice of teaching methodology reform, the feasibility and necessity of implementing hybrid $\mathrm{CBL}+\mathrm{TBL}$ teaching method in the education of internal medicine in TCM universities have been verified, which is of great advantageous to the improvement of students' comprehension, stimulation of their enthusiasm, and enhancement of the teaching validity of internal medicine. The recent study provides reference resources for the teaching reform of medical disciplines in TCM universities in the future.

\section{Competing Interests}

The authors declare that they have no competing interests.

\section{Acknowledgements}

The present study was supported by the Beijing Undergraduate Teaching Reform and Innovation Project and the Key Project of Education and Teaching of Beijing University of Chinese Medicine (XJZX18002).

\section{References}

[1] A. Soffer, M. Siegler, A. R. Tarlov, Internal medicine, Jama 241 (13) (1979) 1363-5.

[2] A. Miller, Z. Fleet, The future of internal medicine: a new curriculum for 2019, British journal of hospital medicine (London, England: 2005) 79 (4) (2018) 225-228.

[3] M. Mancinetti, S. Guttormsen, C. Berendonk, Cognitive load in internal medicine: What every clinical teacher should know about cognitive load theory, European journal of internal medicine 60 (2019) 4-8.

[4] M. Bi, Z. Zhao, J. Yang, Y. Wang, Comparison of case-based learning and traditional method in teaching postgraduate students of medical oncology, Medical teacher 41 (10) (2019) 1124-1128.

[5] W. Zhao, L. He, W. Deng, J. Zhu, A. Su, Y. Zhang, The effectiveness of the combined problem-based learning (PBL) and case-based learning $(\mathrm{CBL})$ teaching method in the clinical practical teaching of thyroid disease, 20 (1) (2020) 381.

[6] C. M. Haley, B. Brown, A. Koerber, C. L. Nicholas, A. Belcher, Comparing Case-Based with Team-Based Learning: Dental Students' Satisfaction, Level of Learning, and Resources Needed, Journal of dental education 84 (4) (2020) 486-494.

[7] C. Ding, S. Li, B. Chen, Effectiveness of flipped classroom combined with team-, case-, lecture- and evidence-based learning on ophthalmology teaching for eight-year program students, 19 (1) (2019) 419.

[8] D. Li, H. Xu, Y. Wang, M. Wei, The Development of Chinese Medical Education, The Journal of craniofacial surgery 31 (2) (2020) 453-455.

[9] J. C. Zheng, H. Zhang, B. Wu, Z. Tong, Y. Zhu, M. S. Mitchell, X. Sun, Y. Yang, K. Zhang, L. Fang, E. Adashi, Medical Education Reform in China: The Shanghai Medical Training Model, Journal of graduate medical education 12 (6) (2020) 655-660.

[10] P. Bintaro, S. Schneidewind, V. Fischer, The development of the internal medicine courses at Hannover Medical School from 2001 to 2018, GMS journal for medical education 36 (5) (2019) Doc56.

[11] R. Faisal, S. Bahadur, L. Shinwari, Problem-based learning in comparison with lecture-based learning among medical students, JPMA. The Journal of the Pakistan Medical Association 66 (6) (2016) 650-3.

[12] A. Alhazmi, M. F. A. Quadri, Comparing case-based and lecture-based learning strategies for orthodontic case diagnosis: A randomized controlled trial, 84 (8) (2020) 857-863.

[13] A. B. Rezende, A. G. F. de Oliveira, T. C. Vale, L. A. S. Teixeira, A. R. A. Lima, A. L. G. Lucchetti, G. Lucchetti, S. H. C. Tibiriçá, O. S. Ezequiel, Comparison of Team-Based Learning versus Traditional Lectures in Neuroanatomy: Medical Student Knowledge and Satisfaction, Anatomical sciences education 13 (5) (2020) 591-601.

[14] H. Liu, X. F. Mi, Z. Z. Huang, B. C. Heng, W. L. Shen, Challenges and strategies in developing team-based learning in Chinese medical education, Medical teacher 42 (11) (2020) 1243-1249. 
[15] R. Elledge, Current thinking in medical education research: an overview, The British journal of oral \& maxillofacial surgery 56 (5) (2018) 380-383.

[16] W. Jiang, Y. Tian, J. Chen, B. Chen, Team-, case-, lecture- and evidence-based learning, Medical education 51 (11) (2017) 1172 .

[17] M. Gartmeier, T. Pfurtscheller, A. Hapfelmeier, M. Grünewald, J. Häusler, T. Seidel, P. O. Berberat, Teacher questions and student responses in case-based learning: outcomes of a video study in medical education, BMC medical education 19 (1) (2019) 455 .
[18] T. Harman, B. Bertrand, A. Greer, A. Pettus, J. Jennings, E. Wall-Bassett, O. T. Babatunde, Case-based learning facilitates critical thinking in undergraduate nutrition education: students describe the big picture, Journal of the Academy of Nutrition and Dietetics 115 (3) (2015) 378-388.

[19] C. L. T. Ho, Team-based learning: A medical student's perspective, Medical teacher 41 (9) (2019) 1087.

[20] M. S. Del Pozo, C. Redondo, Homogenizing the Teaching of Engaged Medical Examinations to Large Numbers of Students: the Experience of the Digital Rectal Examination, (2020). 